\title{
Dexamethasone vs. betamethasone for preterm birth: a systematic review and network meta-analysis
}

\author{
Agustín Ciapponi $^{1}$, Karen Klein ${ }^{1}$, Daniela Colaci ${ }^{1}$, Fernando Althabe ${ }^{2}$, J. M. Belizan ${ }^{1}$, \\ Allie Deegan ${ }^{3}$, Areti Veroniki ${ }^{4}$, and Iván Flórez ${ }^{5,6}$ \\ ${ }^{1}$ 1. Instituto de Efectividad Clínica y Sanitaria (IECS-CONICET) \\ ${ }^{2}$ World Health Organization \\ ${ }^{3}$ New York University College of Global Public Health \\ ${ }^{4}$ University of Ioannina \\ ${ }^{5}$ University of Antioquia \\ ${ }^{6}$ McMaster University Faculty of Health Sciences
}

June 18, 2020

\begin{abstract}
Objective To compare the effectiveness and safety of dexamethasone versus betamethasone for preterm birth (registered in PROSPERO CRD42017078006). Search strategy We searched in MEDLINE, EMBASE, Cochrane Library, LILACS, Clinical Trials.gov, International Clinical Trials Registry Platform, reference lists and contacted field experts. Selection criteria, data collection and analysis Randomized controlled trials comparing any corticosteroids against each other or against placebo. Three researchers independently selected, extracted data and assessed the risk of bias of the included studies by using EROS and COVIDENCE software. We performed a pairwise meta-analysis and Bayesian network meta-analysis. Main results We included 45 trials (11227 women, 11878 infants). There was no important difference between corticosteroids in neonatal death (odds ratio[OR] 1.05; 95\% confidence interval 0.62-1.84; moderate-certainty evidence[CE]), neurodevelopmental disability (OR 1.03; 0.80-1.33; moderate-CE), intraventricular haemorrhage (OR 1.04; 0.56-1.78); low-CE) and birthweight (+5.29 gr; -49.79 to 58.97; high-CE). Compared with betamethasone, dexamethasone may reduce chorioamnionitis (OR 0.70; 0.45-1.06; moderate$\mathrm{CE}$ ), foetal death (OR 0.81; 0.24-2.41; low-CE) while may increase puerperal sepsis (OR 2.04; 0.72-6.06; low-CE) and respiratory distress syndrome (OR 1.34; 0.96-2.11; moderate-CE), however, the confidence interval indicates both beneficial and detrimental effects. Conclusions We found no important difference on neonatal death, neurodevelopmental disability, intraventricular haemorrhage and birthweight between corticosteroids. Compared with betamethasone, dexamethasone may reduce chorioamnionitis and foetal death, but may increase endometritis/puerperal sepsis and respiratory distress syndrome. Further research is warranted to improve the certainty of evidence. Keywords preterm birth, antenatal corticosteroids, dexamethasone, betamethasone, systematic review, network meta-analysis
\end{abstract}

\section{INTRODUCTION}

Preterm birth (less than 37 weeks' gestation) accounts for around $11 \%$ of all live births worldwide, poses risks of adverse outcomes and can be attributed $35 \%$ of deaths among newborns. ${ }^{1-3}$ Preterm birth represents a significant health burden worldwide, mainly in Low-to-Middle-Income Countries (LMICs).

Respiratory distress Syndrome (RDS) is a serious complication of preterm birth and the primary cause of early neonatal death, lifelong disability and poor quality of life. RDS affects up to half of babies born before 28 weeks and a third of babies born before 32 weeks. ${ }^{4}$ Antenatal corticosteroids for preterm birth prevent RDS and neonatal mortality ${ }^{5}$, however there still persist doubts about the applicability in $\mathrm{LMICs}^{6}$ and there is no consensus regarding the type of corticosteroid to use; nor the dose, frequency, timing of use or the route 
of administration. Currently, either betamethasone or dexamethasone are the recommended corticosteroid for clinical practice. The World Health Organization (WHO) guidelines ${ }^{7}$ states that there is no conclusive evidence that would support a recommendation of one over the other. We acknowledged that dexamethasone has an advantage over betamethasone in terms of lower cost and wider availability, and it is currently listed on the WHO Essential Medicine List and in WHO's Managing complications in pregnancy and childbirth guide. $^{8}$

Two Cochrane systematic reviews have synthesized the effects of corticosteroids. Brownfoot et al. $2013^{9}$ and Roberts et al. $2017^{10}$, which compared any corticosteroids for preterm birth against each other, or against placebo, respectively. Although Brownfoot et al. ${ }^{9}$ focused on direct comparisons, authors also assessed indirect comparisons of corticosteroids with placebo for some outcomes based on Roberts $2006^{11}$. While the indirect estimates suggest no significant differences between corticosteroids for puerperal sepsis, a significant difference favoured betamethasone for chorioamnionitis. ${ }^{11}$

Direct comparisons in Brownfoot $2013^{9}$ showed that dexamethasone may have some benefits compared to betamethasone such as less intraventricular haemorrhage. Roberts $2017^{10}$ suggested that dexamethasone may also be associated with a higher rate of chorioamnionitis. New additional published trials ${ }^{12-14}$, that almost doubled the previous number of participants involved in direct comparisons, warranted a network meta-analysis (NMA), to urgently define this hot topic. Our aim was to assess the comparative clinical effectiveness and safety of dexamethasone versus betamethasone for women at risk of preterm birth.

\section{METHODS}

We reported the study according to the PRISMA extension statement for NMA. ${ }^{15}$ The study protocol, registered in PROSPERO (CRD42017078006), contains method details.

\section{Search strategy}

We conducted a literature search strategy without language restriction. We updated the exhaustive searches of the previous Cochrane reviews ${ }^{9,10}$ from the oldest search date reported in Brownfoot $2013^{9}$ (February 13 , 2013) until October 2019, in PubMed MEDLINE, EMBASE, LILACS, Cochrane Library, Clinical Trials.gov and the International Clinical Trials Registry Platform (ICTRP) for ongoing trials search. The MeSH search terms included premature birth, betamethasone, dexamethasone and glucocorticoids (Full search strategy in Appendix S1 ). We also searched references of included studies and contacted experts for additional evidence.

\section{Study selection and data collection}

We included published or unpublished randomized controlled trials (RCT) or quasi-RCT that included women at risk of preterm birth (before 37 weeks), and comparing any corticosteroids against each other or against placebo regardless the dose or schedule.

The primary outcomes for the mother (however defined by study authors) were chorioamnionitis and endometritis/puerperal sepsis, for the foetus/neonate were neonatal death and RDS and for the child neurodevelopmental disability at two years follow-up (blindness, deafness, moderate/severe cerebral palsy, or development delay/intellectual impairment ${ }^{16}$.

The secondary outcomes were: maternal death; perinatal death; foetal death; chronic lung disease; intraventricular haemorrhage; mean birthweight; and low birth weight.

Three authors (KK, DC, AC) independently screened titles and abstracts and reviewed the full-texts of the potentially eligible studies by using EROS ${ }^{17}$ and COVIDENCE $^{18}$ software, and independently extracted data into a pre-piloted data extraction form including the RoB of using the Cochrane tool ${ }^{6}$. We classified a summary RoB for each study as high risk if at least one domain is classified as high risk and the others as low/moderate risk.

\section{Data synthesis and statistical analysis}


We conducted the statistical analyses in accordance with Cochrane guidelines. ${ }^{19}$

We estimated odds ratio (OR) for dichotomous outcomes and mean difference (MD) for continuous outcomes, along with their $95 \%$ confidence intervals (CI) trough pairwise meta-analysis for direct comparisons. Heterogeneity was quantified with the $\mathrm{I}^{2} \operatorname{statistic}^{20}$ (30-60\% was considered 'moderate' heterogeneity). We used a random-effects model and tested subgroup differences $\left(\mathrm{P}<0.05\right.$ or $\left.\mathrm{I}^{2}>30 \%\right)$.

We performed a Bayesian random-effects NMA to estimate treatment effects and $95 \%$ credible intervals (CrI), if the between-study homogeneity, transitivity and coherence assumption across treatment comparisons were judged to be justifiable. ${ }^{21,22,23,24}$ We explored the network geometry and connectivity using network diagrams.

We assessed the statistical heterogeneity of the entire network by the heterogeneity variance $\left(\tau^{2}\right)$ considering the empirical distribution. ${ }^{25}$

Our prespecified subgroup analyses included gestational age at trial entry (24-28, 29-34, 35-37 weeks); intact vs ruptured membranes and country income level: LMIC vs $\mathrm{HIC}^{26}$. We performed sensitivity analysis by low-moderate overall quality of the studies and by using masked treatments. We performed network metaregression based on gestational age at entry, GNI per capita and the year of publication.

We assessed small-study effects and publication bias, ${ }^{27}$ We estimated SUCRA values with their $\mathrm{CrIs}^{28,29}$ in a rank-heat plot. ${ }^{30} \mathrm{NMA}$ were conducted in OpenBugs (version 3.2.3) ${ }^{31}$ and pairwise meta-analysis in RevMan $5.3 .^{32}$.

We assessed the confidence in the estimates by outcome using the GRADE approach and specific criteria for intransitivity (based on potential effect modifiers) and incoherence (based on the statistical consistency). ${ }^{33,34}$ Two authors (AC, IDF) independently graded the certainty of the evidence (CE), and differences were resolved by consensus.

Additionally, we conducted a focus group to reflect patients' perspectives in the discussion (Appendix S3 ) .

\section{RESULTS}

We identified 765 records after removing duplicates, and we finally included $45 \mathrm{RCTs}^{12-14,35-76}$ involving 11,227 women and 11,878 infants (Figure 1). Thirteen RCTs compared dexamethasone vs. betamethasone (2,903 women and 3,170 infants) and 32 compared corticosteroids vs. placebo/no treatment (8,324 women and 8,708 infants). In addition to the 12 trials $^{28,29,31,32,39,44,47,48,54,56,59,62}$ included by Brownfoot $2013^{9}$ and the $30^{21-27,30,33-38,40-43,45,46,49-53,55,57,58,60,61}$ included by Roberts $2017^{10}$, we included three additional ones $^{12-14}$ (1,896 women and 2,059 infants) and we identified four ongoing trials ${ }^{6,77-79}$.

\section{Characteristics of included, excluded and ongoing studies}

The included trials had heterogeneous characteristics and intervention schemes. The most common doses used were $24 \mathrm{mg}$ of dexamethasone and betamethasone (Appendix S2: Table S2.1 ,S2.2 ). The studies were published between 1972 and 2019. Sixteen studies were conducted in the USA, four in Iran, and two studies each were conducted in United Kingdom, the Netherlands, Finland, France, Israel and Brazil. Most studies were conducted in HICs (37), five in upper MICs and only three in lower MICs (median GNI per capita was 20,170 USD). The sample size varied from 18 to 2,831 women (median 118 women). Membranes were intact, ruptured and mixed (both intact and ruptured) in 7, 12 and 26 studies, respectively. Regarding the recruitment gestational age, 30 RCTs set 23-28 weeks as the lower limit and 33 studies set 34-37 weeks as the upper limit (median gestational age 30.44 weeks).

Table S2.3 describes the ongoing studies' characteristics, ${ }^{6,77-79}$ and Table S2.4 the reason of excluded studies initially included by full-text ${ }^{80-82}$ ).

Risk of bias assessment of included studies 
Global RoB was considered low in 21 studies (47\%), $58 \%$ for random sequence generation, $38 \%$ for allocation concealment, $49 \%$ for blinding of participants and personnel, $33 \%$ for blinding of outcome assessment, $56 \%$ for incomplete outcome data, $69 \%$ for selective reporting and $36 \%$ for other bias (Appendix S4: Risk of bias figures and tables ).

\section{NMA results: dexamethasone vs. betamethasone}

The geometry of the treatment networkis presented in Figure $\mathbf{2}$, and the direct, indirect and NMA (mixed) effect estimations for the main eight outcomes of the comparison dexamethasone vs. betamethasone are presented in Figure 3 and Table $\mathbf{1}$ of Summary of finding. We also present in the Appendix S5 the direct comparison forest-plots, in Appendix S6 the summary of finding tables of each corticosteroid vs. control, in Appendix S7 the pairwise meta-analysis forest-plots by type of corticosteroid against placebo and in Appendix S8 the NMA outputs and SUCRA values.

-Chorioamnionitis (6,698 patients, 15 studies)

Compared with placebo/no treatment (control), dexamethasone probably increases chorioamnionitis but in one side of the confidence interval could also reduce it: OR 1.46 (95\% CI 0.81-2.66). On the contrary betamethasone reduces chorioamnionitis: OR 0.63 (95\% CI 0.41-0.95). The test for subgroup differences by corticosteroid type showed this disparity $\left(\mathrm{P}=0.010, \mathrm{I}^{2} 84.2 \%\right)$.

Compared with betamethasone, dexamethasone probably reduces chorioamnionitis but in one side of the CrI is slightly detrimental:OR $\mathbf{0 . 7 0}(95 \% \mathrm{CrI} 0.45-1.06)$, moderate-CE . We found serious incoherence between direct and indirect evidence (Ratio of OR [ROR] 3.18 [95\%CI 1.26-8.02]).

-Endometritis/puerperal sepsis (4,030 patients, 10 studies)

Dexamethasone may increase endometritis/puerperal sepsis and betamethasone probably have little or no effect against control: OR 1.93 (95\% CI 0.8534.41) and 0.94 (95\%CI 0.47-1.87), respectively (Test for subgroup differences suggest disparities; P: 0.16, $\mathrm{I}^{2} 49.8 \%$ ).

There was no report of direct evidence regarding this outcome. Indirect evidence suggest that compared with betamethasone, dexamethasone may increase endometritis/puerperal sepsis, but in one side of the $\mathrm{CrI}$ is protective: OR 2.04 (95\% CrI 0.72-6.06), low-CE .

-Neonatal Death (8697 patients, 23 studies)

Both dexamethasone and betamethasone reduce neonatal death against control: OR 0.60 (95\% CI 0.37-0.94) and OR 0.57 (95\%CI 0.39-0.80), respectively (Test for subgroup differences shows no disparity; P: 0.81, $\mathrm{I}^{2}$ $0 \%$ ). We found no incoherence; ROR 1.15 (95\% CI 0.44-2.96), therefore we considered the NMA evidence the most reliable estimation. Compared with betamethasone, dexamethasone probably has no effect on neonatal death, but the $\mathrm{CrI}$ is compatible with beneficial or detrimental effect:OR 1.05 (95\% CrI 0.62-1.84), moderate-CE .

-Foetal death (3857 patients, 13 studies)

Dexamethasone may reduce foetal death and betamethasone probably have little or no effect against control: OR 0.86 (95\%CI 0.32-2.16) and 1.05 (95\%CI 0.58-2.15), respectively (Test for subgroup differences shows no disparity; P: 0.70, $\mathrm{I}^{2} 0 \%$ ). There was no report of direct evidence regarding this outcome. Indirect evidence suggest that compared with betamethasone, dexamethasone may reduce foetal death, but the CrI limits is compatible with large beneficial or detrimental effect: OR $\mathbf{0 . 8 1}$ (95\% CrI 0.24-2.41), low-CE .

-Respiratory distress Syndrome (9784 patients, 30 studies)

Both dexamethasone and betamethasone may reduce neonatal death against control: OR 0.64 (95\%CI 0.470.90 ) and 0.47 (95\% CI 0.35-0.60), respectively (Test for subgroup differences suggest disparities; P: 0.11, $\mathrm{I}^{2}$ $54.7 \%)$. 
We found no serious incoherence; ROR 1.14 (95\%CI 0.71-2.75), therefore we considered the NMA evidence the most reliable estimation. Compared with betamethasone, dexamethasone probably increases RDS but the CrI is compatible with a small protective effect: OR 1.38 (95\% CrI 0.96-2.11), moderate-CE .

- Neurodevelopmental disability (2628 patients, 3 studies)

We did not find direct evidence for betamethasone vs. placebo. Dexamethasone may reduce neurodevelopmental disability against control: OR 0.39 (95\% CI 0.01-8.08).

Compared with betamethasone, dexamethasone probably has no effect on neurodevelopmental disability, but the CrI is compatible with large beneficial or detrimental effect: OR 1.14 (95\% CrI 0.24-13.86). Two of the included studies had rare events. The frequentist analysis suggested more precise and reliable estimation an OR $\mathbf{1 . 0 3}$ (95\% CI 0.80-1.33), moderate-CE .

-Intraventricular haemorrhage (IVH) (7449 patients, 17 studies)

Both dexamethasone and betamethasone reduce IVH: OR 0.473 (95\%CI $0.281-0.738)$ and 0.381 (95\%CI 0.191-0.668), respectively (Test for subgroup differences shows no disparity; P: 0.88, $\mathrm{I}^{2} 0 \%$ ).

We found no serious incoherence; ROR 1.54 (95\%CI 0.57-4.16). The NMA evidence suggested that compared with betamethasone, dexamethasone may reduce IVH but the CrI is compatible with beneficial or detrimental effect: OR 0.812 (95\% CrI 0.420-1.427), low-CE. However, we found mild heterogeneity ( $\left.\mathrm{I}^{2} 31 \%\right)$ and important subgroup differences by corticosteroid type $\left(\mathrm{I}^{2} 63.5 \%\right)$.

Considering the very high risk of attrition bias ( $43 \%$ of non-analysed infants), the unique marked effect favouring dexamethasone of the study Elimian $2007^{46}$ and the incoherence that this study had generated, we performed a post-hoc sensitivity analysis excluding it. Both the heterogeneity and subgroup differences changed to an $\mathrm{I}^{2}$ of $0 \%$. The new estimation, stilllow-CE, OR 1.04 (95\% CrI 0.56-1.78) was more consistent with the indirect evidence (ROR 1.14 (95\% CI 0.51-2.57) and therefore we considered it as the most reliable estimation (forest-plots in Appendix S5 ).

-Mean birthweight (8645 patients, 23 studies)

Both dexamethasone and betamethasone have no effect on birthweight against control: MD -17.04gr (95\%CI $-75.48 ; 41.41)$ and -9.74 gr. (95\%CI $-43.11 ; 23.63)$, respectively (Test for subgroup differences shows no disparity; P: 0.80, $\mathrm{I}^{2}$ 0\%). We found no serious incoherence; ROR 1.15 (95\% CI 0.44-2.96) and both direct and indirect evidence were considered as high certainty evidence, therefore we considered the NMA evidence the most reliable estimation. Dexamethasone has no effect mean birthweight:mean difference $+5.29 \mathrm{gr}$ $(95 \% \mathrm{CrI}-49.79,58.97)$ high-CE .

\section{-Other outcomes}

We did not find direct evidence about low birthweight. May be no difference between betamethasone vs. control on maternal death: OR 0.98 (95\% CI 0.06-15.90). Dexamethasone and betamethasone probably reduce perinatal death: OR 0.62 (95\%CI 0.33-1.18) and 0.66 (95\%CI 0.48-0.91), respectively (Test for subgroup differences shows no disparity; P: $0.86, \mathrm{I}^{2} 0 \%$ ). Dexamethasone may increase chronic lung disease and betamethasone may reduce this outcome, but the $95 \% \mathrm{CI}$ is also be compatible with large beneficial or detrimental effect: OR 1.30 (95\% CI $0.57-2.96)$ and 0.75 (95\% CI 0.22-2.62), respectively (Test for subgroup differences shows no disparity; P: 0.47, $\mathrm{I}^{2} 0 \%$ ).

We did not find direct evidence regarding maternal death, perinatal death, and we found scarce evidence about low birth weight and chronic lung disease and they were assessed only by pairwise meta-analysis. Compared with betamethasone, dexamethasone may reduce these outcomes, however the $95 \% \mathrm{CI}$ is compatible with large beneficial or detrimental effect: OR 0.75 (95\%CI 0.33-1.71, low-CE) and OR 0.92 (95\% CI 0.62-1.37) respectively (forest-plots in Appendix S5 ).

Meta-regression did not find statistically significant differences. The subgroup and sensitivity analysis did not reveal important changes regarding the main analysis. All the $95 \% \mathrm{CI}$ were compatible with beneficial or 
detrimental effects (Appendix S9).

\section{DISCUSSION}

\section{Main Findings}

Both corticosteroids have proven effective for women at risk of preterm birth on most neonatal and child relevant outcomes compared with placebo or no treatment.

We found that compared with betamethasone, dexamethasone may reduce the rates of chorioamnionitis around $30 \%$ and foetal death $20 \%$, but may increase puerperal sepsis $100 \%$ and respiratory distress syndrome 40\%. Probably, there are no difference in neonatal death and neurodevelopmental disability and may have no difference in IVH and in birthweight. Except for neurodevelopmental disability, and birthweight these effects were imprecise.

\section{Strengths and limitations}

Among the strengths of our work we can mention that we followed the Cochrane guidelines Cochrane ${ }^{19}$, the PRISMA-NMA extension ${ }^{15}$ for reporting and we registered the study protocol in advance. Our work is the most updated and complete systematic review assessing clinical effectiveness and safety of corticosteroids. Our exhaustive search strategy, included clinical trials registries and contacted experts for additional relevant evidence. Although we did not hand-search conference proceedings it is unlikely that our search strategy missed RCTs not included in biomedical databases nor the trials registers.

This NMA added two small trials ${ }^{13,14}$ and one of large good quality trial that compared directly dexamethasone with betamethasone ${ }^{12}$ to the body of evidence. It provided new indirect estimations and increased the precision of the estimations, still low for most outcomes, by combining direct and indirect evidence. The prespecified meta-regression, subgroup and sensitivity analyses reinforced the robustness of our results.

We assessed the certainty of the evidence by the GRADE-NMA approach ${ }^{33,34}$, the validity of the transitivity assumption by comparing the distribution of potential effect modifiers across comparisons and the coherence assumption by the design-by-treatment interaction model and loop-specific approaches. ${ }^{22,24}$

The results of the NMA were mostly coherent, except for chorioamnionitis, may be due to differences between populations included in indirect and direct evidence, and differences in RoB. The indirect evidence came mostly from mothers with ruptured membranes ${ }^{36,37,41,47,49,51,52,55-57,60,65,67,69,72}$ while the direct evidence from a mix of mothers with intact and ruptured membranes ${ }^{12}$. However, meta-regression, subgroup and sensitivity analyses did not explain this incoherence. Therefore, for chorioamnionitis, following the GRADE approach $^{33}$, we considered the direct evidence the most reliable estimation of 23 fewer cases (43 fewer or 5 more) per 1000 women treated with dexamethasone.

We included studies conducted in a range of 50 years and healthcare advances, specifically in neonatology, could be an extra-source of heterogeneity that could partially explain the contradictory direction of effect for some outcomes, but the effect modifiers or RoB did not provide a solid explanation of the effects. The contradictory beneficial or detrimental effect of different outcomes warranted our decision to explore the patients' perspectives about our findings comparing corticosteroids trough a focus group (Appendix 3). Briefly, women failed to make a decision about which corticosteroids they would choose because the trade-off between risk and benefits were very complex for them. They agreed that it would be a decision that they would share or delegate to a professional with whom they established a bond of trust.

The evidence shows limitations, regarding its generalizability to lower-resource countries, since only three ${ }^{14,42,47}(7 \%)$ out of the included RCTs were from lower-MICs and none from LICs. Trials have been largely conducted in tertiary hospitals and recruited highly selected populations. ${ }^{83}$ Concerns about safety and efficacy in low-resource settings were supported by the adverse findings in neonatal deaths and maternal infection of ACT, a community-based, cluster-RCT conducted in six LMICs. ${ }^{84}$ However we did not find important differences by income country classification and by GNI per capita. Hopefully, the ongoing ACTION study could answer this question. ${ }^{6}$ 


\section{Interpretation}

Antenatal corticosteroids for preterm birth have shown to reduce neonatal morbidity and mortality ${ }^{10}$ are cost-effective $^{85}$, and are routinely recommended worldwide. ${ }^{7,86,87}$ The Cochrane review, ${ }^{10}$ and our review are aligned with these studies and recommendations.

The other Cochrane review, ${ }^{9}$ comparing both corticosteroids, did not include recent evidence. The new trial ASTEROID ${ }^{12}$, almost doubled the number of included women under direct comparisons, provided information for our main outcomes, improved the precision for neurosensory disability estimates, and unlike that it was believed it found that dexamethasone may have a beneficial effect on chorioamnionitis.

The potential beneficial effect of dexamethasone on IVH suggested by very low-CE, was reduced with the inclusion of the ASTEROID trial ${ }^{12}$ and completely disappeared when excluding Elimian $2007 .{ }^{46}$ This posthoc sensitivity analysis, based on the very high risk of attrition bias of this study, provided more consistent results with the indirect evidence. Additionally, a meta-analyses found an increased risk of neurodevelopmental impairment in children with periventricular/intraventricular haemorrhage ${ }^{88}$, mainly driven by cerebral palsy $^{89}$. Since we did not find a differential effect of dexamethasone on neurosensory disability it would be unlikely a favourable effect on IVH. Additionally, even if a reduction in IVH was true, it is more important the observed absence of differences on long-term disability for the quality of life of survivors. ${ }^{90}$

Roberts $2006^{11}$ assessed indirect estimations favouring betamethasone for chorioamnionitis. This was consistent with our indirect estimation but opposite to the ASTEROID trial ${ }^{12}$ findings that were considered the most reliable estimation for this outcome.

Our NMA improved the precision and certainty of most previous estimations. We identified a another NMA that evaluated antenatal maternal administration dexamethasone, betamethasone and ambroxol to prevent RDS. ${ }^{91}$ Compared with placebo, all interventions reduced RDS and neonatal death, but no significant difference in the incidence of bronchopulmonary dysplasia. They also suggest that ambroxol seems to be the most effective treatment for reducing the incidence of RDS and neonatal death based on its SUCRA values. This conclusion was not consistent with a Cochrane review ${ }^{92}$ or the relevant preterm birth management guidelines. ${ }^{7,86}$

A wise choice should consider all factors besides evidence, including local availability, costs and costutility. ${ }^{93,94} \mathrm{~A}$ full course of betamethasone costs around US $\$ 35$ while dexamethasone $\$ 1$ (3\% of the cost of betamethasone). ${ }^{94}$ The cost-effectiveness of the administration of betamethasone based in individual trials is controversial, and it should be based in the best estimation of effectiveness. ${ }^{95,96}$ Mainly LMICs still have significant challenges to provide safe and effective antenatal corticosteroid use, including ensuring accurate gestational age determination, establishing clear treatment guidelines, strengthening provider capacity, incorporating corticosteroid in national essential medicines lists, and monitoring use and outcomes. ${ }^{97}$

\section{CONCLUSION}

This comprehensive NMA confirmed that corticosteroids were mostly effective for neonatal and child relevant outcomes compared with placebo or no treatment. There was no important difference between corticosteroids on neonatal death, neurodevelopmental disability, IVH and birthweight. Low to moderate-CE suggest that dexamethasone may reduce chorioamnionitis, and foetal death but may increase puerperal sepsis and RDS. However, the 95\% CI indicates both beneficial and detrimental effects for these outcomes. The opposing direction of these outcomes does not allow to derive recommendations about what corticosteroid should be used and large well designed RCTs are warranted to improve the certainty of evidence. Ideally, they should represent low resource settings and also evaluate the best schemes of administration. Individual participant data meta-analysis could help to answer these questions. In the meantime, monitoring short-term and longterm health outcomes, including neurodevelopmental disability will be important.

Since there is no robust evidence on which corticosteroid should be prescribed, decisions should be based on availability, costs, opportunity, and facilities. Shared decision-making would help patients to take their choices when facing this scenario. 


\section{Disclosure of interests}

All authors have completed the ICMJE uniform disclosure form at www.icmje.org/coi_disclosure.pdf and declare: no support from any organisation for the submitted work; no financial relationships with any organisations that might have an

interest in the submitted work in the previous three years; no other relationships or activities that could appear to have influenced the submitted work.

\section{Contribution to authorship}

JB, FA and AC conceived the study; AC, FA, JB, IDF, AAV designed the study;

KK, DC, AD and AC collected and abstracted the data; IDF, AAV undertook the statistical

analysis; AC, FA, JB, IDF, AAV, KK, AD, DC drafted the manuscript; all authors had full access to all the data, including statistical reports and tables; all authors analysed and interpreted the data; all authors critically revised the manuscript for important intellectual content; AC is the guarantor.

\section{Details of ethics approval}

Not applicable.

\section{Funding and competing interest.}

This study received no external funding.

\section{Acknowledgments}

We acknowledge Daniel Comandé for his support as librarian and Ioannis Gallos and Argyro Papadopoulou for their exhaustive revision of the manuscript.

We also acknowledge the Lic. María Belizán and Lic. Natali Ini for coordinating and analysing the focus group that explored the women's perspectives of our findings.

\section{Supporting Information}

Additional supporting information may be found online in the Supporting Information section at the end of the article.

Appendix S1 : Search Strategy

Appendix S2 : Characteristics of included, ongoing and excluded studies

Table S2.1 Main characteristics of population and setting of included studiesTable S2.2 Characteristics of included studiesTable S2.3 Characteristics of ongoing studiesTable S2.4 Characteristics excluded studies

Appendix S3 : Focus group: patients' perspectives

Appendix S4 : Risk of bias figures and tables

Figure S4.1: Risk of bias graph by risk of bias item (\% across all included studies)Figure S4.2: Risk of bias summary by risk of bias item for each included study

Table S4.1 Support for judgement of included studies by risk of bias item

Appendix S5 : Pairwise meta-analysis results for direct comparison dexamethasone vs. betamethasone forest-plots

Appendix S6 : Summary of finding tables of corticosteroid vs. placebo/no treatment

Table S6.1 Summary of finding table: dexamethasone vs. controlTable S6.2 Summary of finding table: betamethasone vs. control 
Appendix S7 : Pairwise meta-analysis results for direct comparison corticosteroids vs. placebo/no treatment forest-plots by type of corticosteroid

Appendix S8 : Network meta-analysis outputs

Appendix S9 : Meta-regression, subgroup and sensitivity analysis

Appendix S10 : PRISMA NMA Checklist

Table/Figure Caption List

Table 1. Summary of finding table: dexamethasone vs. betamethasone

Figure 1. Study flowchart

Figure 2. Network composition by outcome

Figure 3. Network and direct forest plot for dexamethasone vs. betamethasone

\section{References}

1. Chawanpaiboon S, Vogel JP, Moller AB, Lumbiganon P, Petzold M, Hogan D, et al. Global, regional, and national estimates of levels of preterm birth in 2014: a systematic review and modelling analysis. Lancet Glob Health. 2019 Jan;7(1):e37-e46.

2. Platt MJ. Outcomes in preterm infants. Public Health. 2014 May;128(5):399-403.

3. UN Inter-agency Group for Child Mortality Estimation. Levels and trends in child mortality: report 2017, estimates developed by the UN Inter-agency Group for child mortality estimation. . New York: United Nations Children's Fund; 2017.

4. Glass HC, Costarino AT, Stayer SA, Brett CM, Cladis F, Davis PJ. Outcomes for extremely premature infants. Anesth Analg. 2015 Jun;120(6):1337-51.

5. Crowley P. Prophylactic corticosteroids for preterm birth. Cochrane Database Syst Rev. 2000(2):CD000065.

6. Collaborators WAT. The World Health Organization ACTION-I (Antenatal CorTicosteroids for Improving Outcomes in preterm Newborns) Trial: a multi-country, multi-centre, two-arm, parallel, double-blind, placebo-controlled, individually randomized trial of antenatal corticosteroids for women at risk of imminent birth in the early preterm period in hospitals in low-resource countries. Trials. 2019 Aug 16;20(1):507.

7. WHO. Recommendations on Interventions to Improve Preterm Birth Outcomes. Evidence and recommendations 2015 [cited 2018 12/2018]; Available from:https://www.who.int/reproductivehealth/publications/maternal_perinatal_health/preterm-birthguideline/en/

8. Managing complications in pregnancy and childbirth: a guide for midwives and doctors. 2nd ed. Geneva: World Health Organization; 2017.

9. Brownfoot FC, Gagliardi DI, Bain E, Middleton P, Crowther CA. Different corticosteroids and regimens for accelerating fetal lung maturation for women at risk of preterm birth. Cochrane Database Syst Rev. 2013 Aug 29(8):CD006764.

10. Roberts D, Brown J, Medley N, Dalziel SR. Antenatal corticosteroids for accelerating fetal lung maturation for women at risk of preterm birth. Cochrane Database Syst Rev. 2017 Mar 21;3:CD004454.

11. Roberts D, Dalziel S. Antenatal corticosteroids for accelerating fetal lung maturation for women at risk of preterm birth. Cochrane Database Syst Rev. 2006 Jul 19(3):CD004454. 
12. Crowther CA, Ashwood P, Andersen CC, Middleton PF, Tran T, Doyle LW, et al. Maternal intramuscular dexamethasone versus betamethasone before preterm birth (ASTEROID): a multicentre, double-blind, randomised controlled trial. Lancet Child Adolesc Health. 2019 Sep 12.

13. Mirzamoradi M, Hasani Nejhad F, Jamali R, Heidar Z, Bakhtiyari M. Evaluation of the effect of antenatal betamethasone on neonatal respiratory morbidities in late preterm deliveries (34-37 weeks). The journal of maternal-fetal \& neonatal medicine : the official journal of the European Association of Perinatal Medicine, the Federation of Asia and Oceania Perinatal Societies, the International Society of Perinatal Obstetricians. 2019:1-8.

14. Ontela V, Dorairajan G, Bhat VB, Chinnakali P. Effect of Antenatal Steroids on Respiratory Morbidity of Late Preterm Newborns: A Randomized Controlled Trial. Journal of Tropical Pediatrics. 2018;64(6):531-8.

15. Hutton B, Salanti G, Caldwell DM, Chaimani A, Schmid CH, Cameron C, et al. The PRISMA extension statement for reporting of systematic reviews incorporating network meta-analyses of health care interventions: checklist and explanations. Ann Intern Med. 2015 Jun 2;162(11):777-84.

16. Binder LM, Iverson GL, Brooks BL. To Err is Human: "Abnormal" Neuropsychological Scores and Variability are Common in Healthy Adults. Archives of Clinical Neuropsychology. 2009;24(1):31-46.

17. Ciapponi A, Glujovsky D, Bardach A, García Martí S, Comande D. EROS: a new software for early stage of systematic reviews. HTAi 2011 Conference; 2011 June 27-29, 2011; Rio de Janeiro: Health Technology Assessment International (HTAi); 2011.

18. Covidence systematic review software. Melbourne, Australia: Veritas Health Innovation.

19. Chaimani A, Caldwell DM, Li T, Higgins JP, Salanti G. Chapter 11: Undertaking network meta-analyses. In: Higgins J, Thomas J, Chandler J, MS. C, Li T, Page M, et al., editors. Cochrane Handbook for Systematic Reviews of Interventions Version 6 [updated September 2018]

Cochrane; 2018.

20. Higgins JP, Thompson SG. Quantifying heterogeneity in a meta-analysis. Stat Med. 2002 Jun 15;21(11):1539-58.

21. Baker SG, Kramer BS. The transitive fallacy for randomized trials: if A bests B and B bests C in separate trials, is A better than C? BMC Med Res Methodol. 2002 Nov 13;2:13.

22. Higgins JP, Jackson D, Barrett JK, Lu G, Ades AE, White IR. Consistency and inconsistency in network meta-analysis: concepts and models for multi-arm studies. Res Synth Methods. 2012 Jun;3(2):98-110.

23. Veroniki AA, Vasiliadis HS, Higgins JP, Salanti G. Evaluation of inconsistency in networks of interventions. Int J Epidemiol. 2013 Feb;42(1):332-45.

24. Dias S, Welton NJ, Caldwell DM, Ades AE. Checking consistency in mixed treatment comparison metaanalysis. Stat Med. 2010 Mar 30;29(7-8):932-44.

25. Turner RM, Davey J, Clarke MJ, Thompson SG, Higgins JP. Predicting the extent of heterogeneity in meta-analysis, using empirical data from the Cochrane Database of Systematic Reviews. Int J Epidemiol. 2012 Jun;41(3):818-27.

26. World Bank Country and Lending Groups. 2019 [cited 10.20/2019; Available from:https://datahelpdesk.worldbank.org/knowledgebase/articles/906519-world-bank-country-and-lendinggroups

27. Chaimani A, Higgins JP, Mavridis D, Spyridonos P, Salanti G. Graphical tools for network meta-analysis in STATA. PLoS One. 2013;8(10):e76654.

28. Trinquart L, Attiche N, Bafeta A, Porcher R, Ravaud P. Uncertainty in Treatment Rankings: Reanalysis of Network Meta-analyses of Randomized Trials. Ann Intern Med. 2016 May 17;164(10):666-73. 
29. Veroniki AA, Straus SE, Rucker G, Tricco AC. Is providing uncertainty intervals in treatment ranking helpful in a network meta-analysis? J Clin Epidemiol. 2018 Aug;100:122-9.

30. Veroniki AA, Straus SE, Fyraridis A, Tricco AC. The rank-heat plot is a novel way to present the results from a network meta-analysis including multiple outcomes. J Clin Epidemiol. 2016 Aug;76:193-9.

31. Lunn DJ, Thomas A, Best N, Spiegelhalter D. WinBUGS - A Bayesian modelling framework: Concepts, structure, and extensibility. Statistics and Computing. 2000 2000/10/01;10(4):325-37.

32. Review Manager (RevMan). [Computer program] Version 53 Copenhagen: The Nordic Cochrane Centre, The Cochrane Collaboration; 2014.

33. Puhan MA, Schunemann HJ, Murad MH, Li T, Brignardello-Petersen R, Singh JA, et al. A GRADE Working Group approach for rating the quality of treatment effect estimates from network meta-analysis. BMJ. 2014;349:g5630.

34. Brignardello-Petersen R, Bonner A, Alexander PE, Siemieniuk RA, Furukawa TA, Rochwerg B, et al. Advances in the GRADE approach to rate the certainty in estimates from a network meta-analysis. J Clin Epidemiol. 2018 Jan;93:36-44.

35. Effect of antenatal dexamethasone administration on the prevention of respiratory distress syndrome. Am J Obstet Gynecol. 1981 Oct 1;141(3):276-87.

36. Amorim MMR, Santosa LC, Faúndes A. Corticosteroid therapy for prevention of respiratory distress syndrome in severe preeclampsia. American Journal of Obstetrics and Gynecology. 1999 1999/05;180(5):12838 .

37. Attawattanakul N, Tansupswatdikul P. Effects of antenatal dexamethasone on respiratory distress in late preterm infant: a randomized controlled trial. hai Journal of Obstetrics and Gynaecology. 2015;23(1):25-33.

38. Balci O, Ozdemir S, Mahmoud AS, Acar A, Colakoglu MC. The Effect of Antenatal Steroids on Fetal Lung Maturation between the 34th and 36th Week of Pregnancy. Gynecologic and Obstetric Investigation. 2010;70(2):95-9.

39. Block MF, Kling OR, Crosby WM. Antenatal glucocorticoid therapy for the prevention of respiratory distress syndrome in the premature infant. Obstet Gynecol. 1977 Aug;50(2):186-90.

40. Cararach V, Botet F, Sentis J, Carmona F. A multicenter, prospective and randomized study in premature rupture of membranes (PROM). Maternal and perinatal complications. International journal of gynaecology and obstetrics. 1991;36 Suppl:267.

41. Carlan SJ, Parsons M, O'Brien WF, Krammer J. Pharmacologic pulmonary maturation in preterm premature rupture of membranes. American journal of obstetrics and gynecology. 1991;164:371.

42. Chen C-Y, Wang K-G, Chang T-Y, Chen C-P, Loo J-H. Effects of Antenatal Betamethasone and Dexamethasone in Preterm Neonates. Taiwanese Journal of Obstetrics and Gynecology. 2005 2005/09/01/;44(3):247-51.

43. Danesh A, Janghorbani M, Khalatbari S. Effects of antenatal corticosteroids on maternal serum indicators of infection in women at risk for preterm delivery: A randomized trial comparing betamethasone and dexamethasone. J Res Med Sci. 2012 Oct;17(10):911-7.

44. Doran TA, Swyer P, MacMurray B, Mahon W, Enhorning G, Bernstein A, et al. Results of a double-blind controlled study on the use of betamethasone in the prevention of respiratory distress syndrome. American Journal of Obstetrics and Gynecology. 1980 1980/02;136(3):313-20.

45. Egerman RS, Walker RA, Mercer BM, Doss JL, Sibai BM, Andersen RA. Comparison between oral and intramuscular dexamethasone in suppressing unconjugated estriol levels during the third trimester. Am J Obstet Gynecol. 1998 Nov;179(5):1234-6. 
46. Elimian A, Garry D, Figueroa R, Spitzer A, Wiencek V, Quirk JG. Antenatal betamethasone compared with dexamethasone (betacode trial): a randomized controlled trial. Obstet Gynecol. 2007 Jul;110(1):26-30.

47. Fekih M, Chaieb A, Sboui H, Denguezli W, Hidar S, Khairi H. [Value of prenatal corticotherapy in the prevention of hyaline membrane disease in premature infants. Randomized prospective study]. Tunis Med. 2002 May;80(5):260-5.

48. Gamsu HR, Mullinger BM, Donnai P, Dash CH. Antenatal administration of betamethasone to prevent respiratory distress syndrome in preterm infants: report of a UK multicentre trial. British journal of obstetrics and gynaecology. 1989;96(4):401-10.

49. Garite TJ, Rumney PJ, Briggs GG, Harding JA, Nageotte MP, Towers CV, et al. A randomized, placebo-controlled trial of betamethasone for the prevention of respiratory distress syndrome at 24 to 28 week's gestation. American Journal of Obstetrics and Gynecology. 1992 1992/02;166(2):646-51.

50. Goodner DM. Antenatal steroids in the treatment of respiratory distress syndrome. 9th world congress of gynecology and obstetrics; 1979 october 26-31; tokyo, japan. 1979:362.

51. Gyamfi-Bannerman C, Thom EA, Blackwell SC, Tita AT, Reddy UM, Saade GR, et al. Antenatal Betamethasone for Women at Risk for Late Preterm Delivery. New England journal of medicine. 2016;374(14):1311-20.

52. Kari MA, Hallman M, Eronen M, Teramo K, Virtanen M, Koivisto M, et al. Prenatal dexamethasone treatment in conjunction with rescue therapy of human surfactant: a randomized placebo-controlled multicenter study. Pediatrics. 1994;93(5):730-6.

53. Khandelwal M, Chang E, Hansen C, Hunter K, Milcarek B. Betamethasone dosing interval: 12 or 24 hours apart? A randomized, noninferiority open trial. American Journal of Obstetrics \& Gynecology. 2012;206(3):201.e1-.e11.

54. Khazardoust S, Javadian P, Salmanian B, Zandevakil F, Abbasalizadeh F, Alimohamadi S, et al. A clinical randomized trial on endocervical inflammatory cytokines and betamethasone in prime-gravid pregnant women at risk of preterm labor. Iranian journal of immunology. 2012;9(3):199-207.

55. Lewis D, Brody K, Edwards M, Brouillette R, Burlison S, London S. Preterm premature ruptured membranes: A randomized trial of steroids after treatment with antibiotics. Obstetrics \& Gynecology. 1996 1996/11;88(5):801-5.

56. Liggins GC, Howie RN. A controlled trial of antepartum glucocorticoid treatment for prevention of the respiratory distress syndrome in premature infants. Pediatrics. 1972;50:515-25.

57. Lopez L, Rojas L, Rodriguez V, Sanchez J. Use of corticoids in preterm pregnancy with premature rupture of membranes. Revista colombiana de obstetricia y ginecologia. 1989;40:147-51.

58. Magee LA, Dawes GS, Moulden M, Redman CW. A randomised controlled comparison of betamethasone with dexamethasone: effects on the antenatal fetal heart rate. Br J Obstet Gynaecol. 1997 Nov;104(11):12338 .

59. Mansouri M, Seyedolshohadaei F, Company F, Setare S, Mazhari S. Effect of antenatal Betamethasone on prevention of respiratory distress syndrome among neonates with gestational age of 35-36 weeks. Journal of Gorgan University of Medical Sciences. 2010;12(3):Pe18-Pe23, En109.

60. Morales WJ, Angel JL, O'Brien WF, Knuppel RA. Use of ampicillin and corticosteroids in premature rupture of membranes: a randomized study. Obstetrics and gynecology. 1989;73(5 Pt 1):721-6.

61. Mulder EJ, Derks JB, Visser GH. Antenatal corticosteroid therapy and fetal behaviour: a randomised study of the effects of betamethasone and dexamethasone. Br J Obstet Gynaecol. 1997 Nov;104(11):1239-47. 
62. Mushkat Y, Ascher-Landsberg J, Keidar R, Carmon E, Pauzner D, David MP. The effect of betamethasone versus dexamethasone on fetal biophysical parameters. Eur J Obstet Gynecol Reprod Biol. 2001 Jul;97(1):50-2.

63. Nelson LH, Meis PJ, Hatjis CG, Ernest JM, Dillard R, Schey HM. Premature rupture of membranes: a prospective, randomized evaluation of steroids, latent phase, and expectant management. Obstetrics and gynecology. 1985;66(1):55-8.

64. Parsons MT, Sobel D, Cummiskey K, Constantine L, Roitman J. Steroid, antibiotic and tocolytic vs no steroid, antibiotic and tocolytic management in patients with preterm PROM at 25-32 weeks. Proceedings of 8th annual meeting of the society of perinatal obstetricians; 1988 feb 3-6; las vegas, nevada, USA. 1988:44.

65. Pattinson RC, Makin JD, Funk M, Delport SD, Macdonald AP, Norman K, et al. The use of dexamethasone in women with preterm premature rupture of membranes-a multicentre, double-blind, placebocontrolled, randomised trial. Dexiprom Study Group. South African medical journal. 1999;89(8):865-70.

66. Porto AMF, Coutinho IC, Correia JB, Amorim MMR. Effectiveness of antenatal corticosteroids in reducing respiratory disorders in late preterm infants: randomised clinical trial. BMJ. 2011 2011/04/12;342(apr12 1):d1696-d.

67. Qublan HS, Malkawi HY, Hiasat MS, Hindawi IM, Al-Taani MI, Abu-Khait SA, et al. The effect of antenatal corticosteroid therapy on pregnancies complicated by premature rupture of membranes. Clinical and experimental obstetrics \& gynecology. 2001;28(3):183-6.

68. Rotmensch S, Liberati M, Vishne TH, Celentano C, Ben-Rafael Z, Bellati U. The effect of betamethasone and dexamethasone on fetal heart rate patterns and biophysical activities. A prospective randomized trial. Acta Obstet Gynecol Scand. 1999 Jul;78(6):493-500.

69. Schutte MF, Treffers PE, Koppe JG, Breur W. The influence of betamethasone and orciprenaline on the incidence of respiratory distress syndrome in the newborn after preterm labour. British journal of obstetrics and gynaecology. 1980;87(2):127-31.

70. Senat MV, Minoui S, Multon O, Fernandez H, Frydman R, Ville Y. Effect of dexamethasone and betamethasone on fetal heart rate variability in preterm labour: a randomised study. Br J Obstet Gynaecol. $1998 \mathrm{Jul} ; 105(7): 749-55$.

71. Shanks A, Gross G, Shim T, Allsworth J, Sadovsky Y, Bildirici I. Administration of steroids after 34 weeks of gestation enhances fetal lung maturity profiles. American Journal of Obstetrics and Gynecology. 2010 2010/07/01/;203(1):47.e1-.e5.

72. Silver RK, Vyskocil C, Solomon SL, Ragin A, Neerhof MG, Farrell EE. Randomized trial of antenatal dexamethasone in surfactant-treated infants delivered before 30 weeks' gestation. Obstetrics and gynecology. 1996;87(5 Pt 1):683-91.

73. Subtil D, Tiberghien P, Devos P, Therby D, Leclerc G, Vaast P, et al. Immediate and delayed effects of antenatal corticosteroids on fetal heart rate: a randomized trial that compares betamethasone acetate and phosphate, betamethasone phosphate, and dexamethasone. Am J Obstet Gynecol. 2003 Feb;188(2):524-31.

74. Taeusch HW, Frigoletto F, Kitzmiller J, Avery ME, Hehre A, Fromm B, et al. Risk of respiratory distress syndrome after prenatal dexamethasone treatment. Pediatrics. 1979;63(1):64-72.

75. Teramo K, Hallman M, Raivio KO. Maternal Glucocorticoid in Unplanned Premature Labor. Controlled Study on the Effects of Betamethasone Phosphate on the Phospholipids of the Gastric Aspirate and on the Adrenal Cortical Function of the Newborn Infant. Pediatric Research. 1980 1980/04;14(4):326-9.

76. Urban R, Lemancewicz A, Przepiesc J, Urban J, Kretowska M. Antenatal corticosteroid therapy: a comparative study of dexamethasone and betamethasone effects on fetal Doppler flow velocity waveforms. Eur J Obstet Gynecol Reprod Biol. 2005 Jun 1;120(2):170-4. 
77. Hong S, Lee SM, Kwak DW, Lee J, Kim SY, Oh JW, et al. Effects of antenatal corticosteroids in twin neonates with late preterm birth (ACTWIN [Antenatal Corticosteroids in TWIN late preterm neonates] trial): study protocol for a randomized controlled trial. BMC Pregnancy Childbirth. 2019 Apr 3;19(1):114.

78. 03547791 N. Effects of ACS in Twin With LPB: study Protocol for a RCT.Https://clinicaltrialsgov/show/nct03547791. 2018.

79. 03446937 N. Effect of Antenatal Corticosteroids on Neonatal Morbidity.Https://clinicaltrialsgov/show/nct03446937. 2018.

80. Cartwright R, Crowther C, Anderson P, Harding J, Doyle L, McKinlay C. Influence of fetal growth restriction on neurocognitive function after repeat antenatal betamethasone: secondary analysis of a randomised trial. Journal of paediatrics and child health. 2019;55:12-3.

81. Cartwright RD, Crowther CA, Anderson PJ, Harding JE, Doyle LW, McKinlay CJD. Association of Fetal Growth Restriction With Neurocognitive Function After Repeated Antenatal Betamethasone Treatment vs Placebo: Secondary Analysis of the ACTORDS Randomized Clinical Trial. JAMA network open. 2019;2(2):e187636.

82. group Bs, the G, Schmitz T, Alberti C, Aupiais C, Ursino M, et al. Full versus half dose of antenatal betamethasone to prevent severe neonatal respiratory distress syndrome associated with preterm birth: study protocol for a randomised, multicenter, double blind, placebo-controlled, non-inferiority trial (BETADOSE). BMC Pregnancy \& Childbirth. 2019;19(1):67-.

83. Vogel JP, Oladapo OT, Pileggi-Castro C, Adejuyigbe EA, Althabe F, Ariff S, et al. Antenatal corticosteroids for women at risk of imminent preterm birth in low-resource countries: the case for equipoise and the need for efficacy trials. BMJ Glob Health. 2017;2(3):e000398.

84. Althabe F, Belizan JM, McClure EM, Hemingway-Foday J, Berrueta M, Mazzoni A, et al. A populationbased, multifaceted strategy to implement antenatal corticosteroid treatment versus standard care for the reduction of neonatal mortality due to preterm birth in low-income and middle-income countries: the ACT cluster-randomised trial. Lancet. 2015 Feb 14;385(9968):629-39.

85. Lee VR, Kaimal AJ, Caughey AB. Cost-effectiveness of antenatal late preterm steroids. American Journal of Obstetrics and Gynecology. 2017;216(1):S233.

86. National Institute for Health and Care Excellence. NICE guideline 25: preterm labour and birth. August, 2019. 2019 [cited December 6, 2019]; Available from:https://www.nice.org.uk/guidance/ng25

87. Committee on Obstetric P. Committee Opinion No. 713: Antenatal Corticosteroid Therapy for Fetal Maturation. Obstet Gynecol. 2017 Aug;130(2):e102-e9.

88. Mukerji A, Shah V, Shah PS. Periventricular/Intraventricular Hemorrhage and Neurodevelopmental Outcomes: A Meta-analysis. Pediatrics. 2015 Dec;136(6):1132-43.

89. Gotardo JW, Volkmer NFV, Stangler GP, Dornelles AD, Bohrer BBA, Carvalho CG. Impact of periintraventricular haemorrhage and periventricular leukomalacia in the neurodevelopment of preterms: A systematic review and meta-analysis. PLoS One. 2019;14(10):e0223427.

90. Saigal S, Doyle LW. An overview of mortality and sequelae of preterm birth from infancy to adulthood. Lancet. 2008 Jan 19;371(9608):261-9.

91. Zhang H, Liu J, Liu T, Wang Y, Dai W. Antenatal maternal medication administration in preventing respiratory distress syndrome of premature infants: A network meta-analysis. Clinical Respiratory Journal. 2018;12(10):2480-90.

92. Gonzalez Garay AG, Reveiz L, Velasco Hidalgo L, Solis Galicia C. Ambroxol for women at risk of preterm birth for preventing neonatal respiratory distress syndrome. Cochrane Database Syst Rev. 2014 Oct 31(10):CD009708. 
93. Vogel JP, Souza JP, Gulmezoglu AM, Mori R, Lumbiganon P, Qureshi Z, et al. Use of antenatal corticosteroids and tocolytic drugs in preterm births in 29 countries: an analysis of the WHO Multicountry Survey on Maternal and Newborn Health. Lancet. 2014 Nov 22;384(9957):1869-77.

94. UN Commission, Born Too soon Care Antenatal Corticosteroids Working Group. Dexamethasone versus betamethasone as an antenatal corticosteroid (ACS). Aug 20, 2013. 2013 [cited Dec 6, 2019]; Available from:https://www.healthynewbornnetwork.org/hnn-content/uploads/ACS-Beta-vs-Dexa-130820.pdf

95. Gyamfi-Bannerman C, Zupancic JAF, Sandoval G, Grobman WA, Blackwell SC, Tita ATN, et al. Cost-effectiveness of Antenatal Corticosteroid Therapy vs No Therapy in Women at Risk of Late Preterm Delivery: A Secondary Analysis of a Randomized Clinical Trial. JAMA Pediatr. 2019 May 1;173(5):462-8.

96. Rosenbloom JI, Lewkowitz AK, Sondgeroth KE, Hudson JL, Macones GA, Cahill AG, et al. Antenatal corticosteroid administration in late-preterm gestations: a cost-effectiveness analysis. Journal of MaternalFetal \& Neonatal Medicine. 2018;31(22):1-7.

97. Greensides D, Robb-McCord J, Noriega A, Litch JA. Antenatal Corticosteroids for Women at Risk of Imminent Preterm Birth in 7 sub-Saharan African Countries: A Policy and Implementation Landscape Analysis. Global health, science and practice. 2018;6(4):644-56.

\section{Hosted file}

Table 1. Summary of finding table. dexamethasone vs. betamethasone.docx available at https://authorea.com/users/333502/articles/460561-dexamethasone-vs-betamethasone-forpreterm-birth-a-systematic-review-and-network-meta-analysis

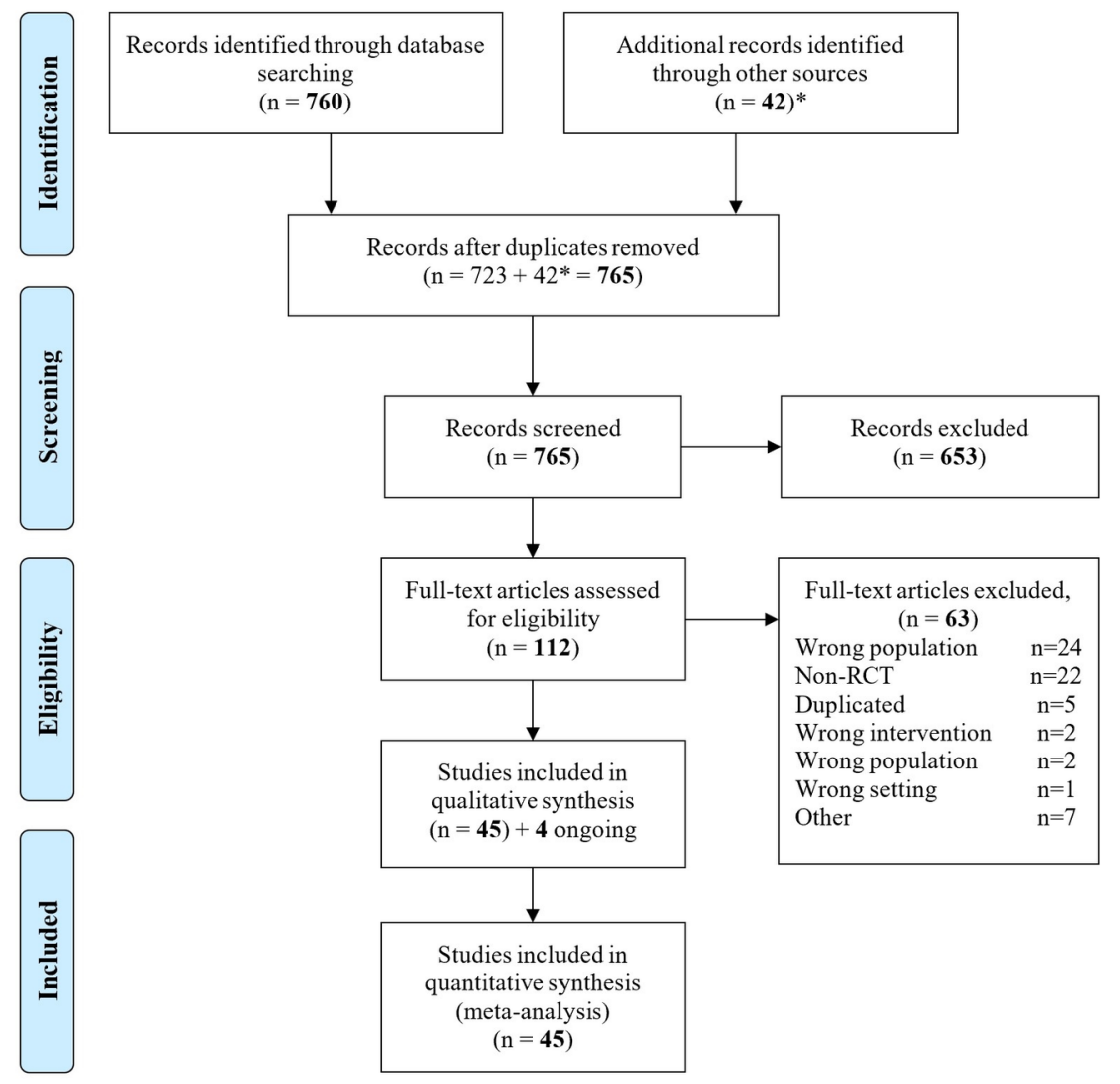


Chorioamnionitis: (6698 patients, 15 studies)

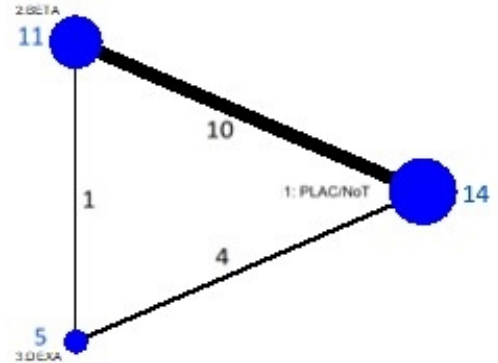

Neonatal Death (8697 patients, 23 studies)

17

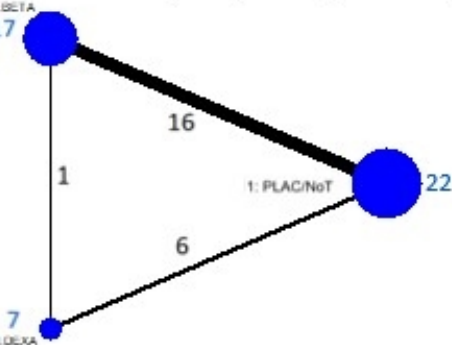

RDS (9784 patients, 30 studies)

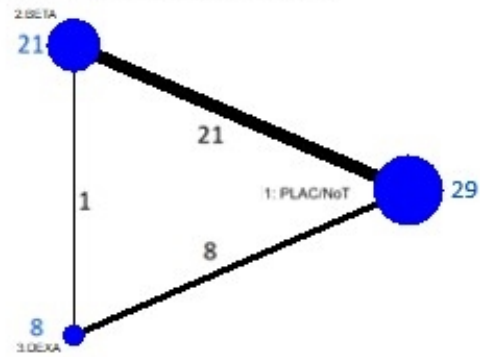

IVH (7449 patients, 17 studies)

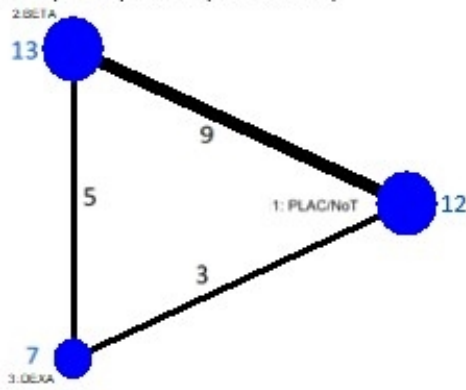

Endometritis (4030 patients, 10 studies)

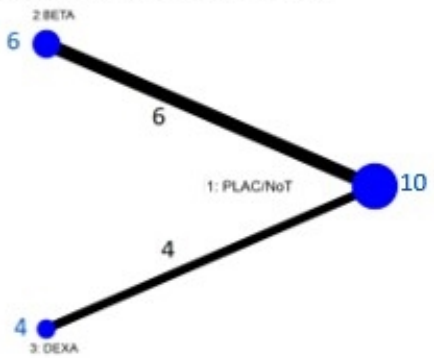

Fetal death (3857 patients, 13 studies)

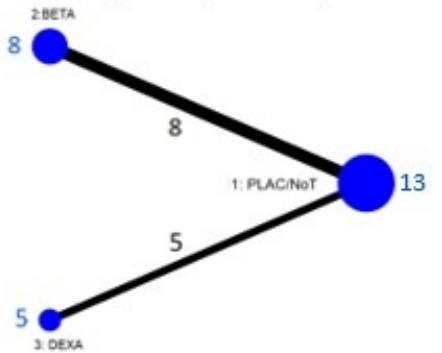

Neurodevelop. disability ( 2628 patients, 3 studies)

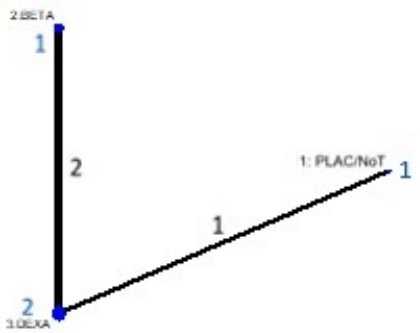

Mean birthweight ( 8645 patients, 23 studies)

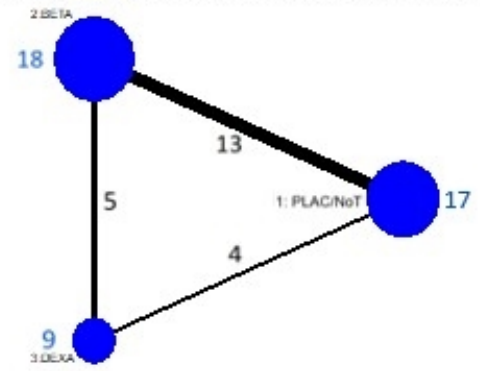




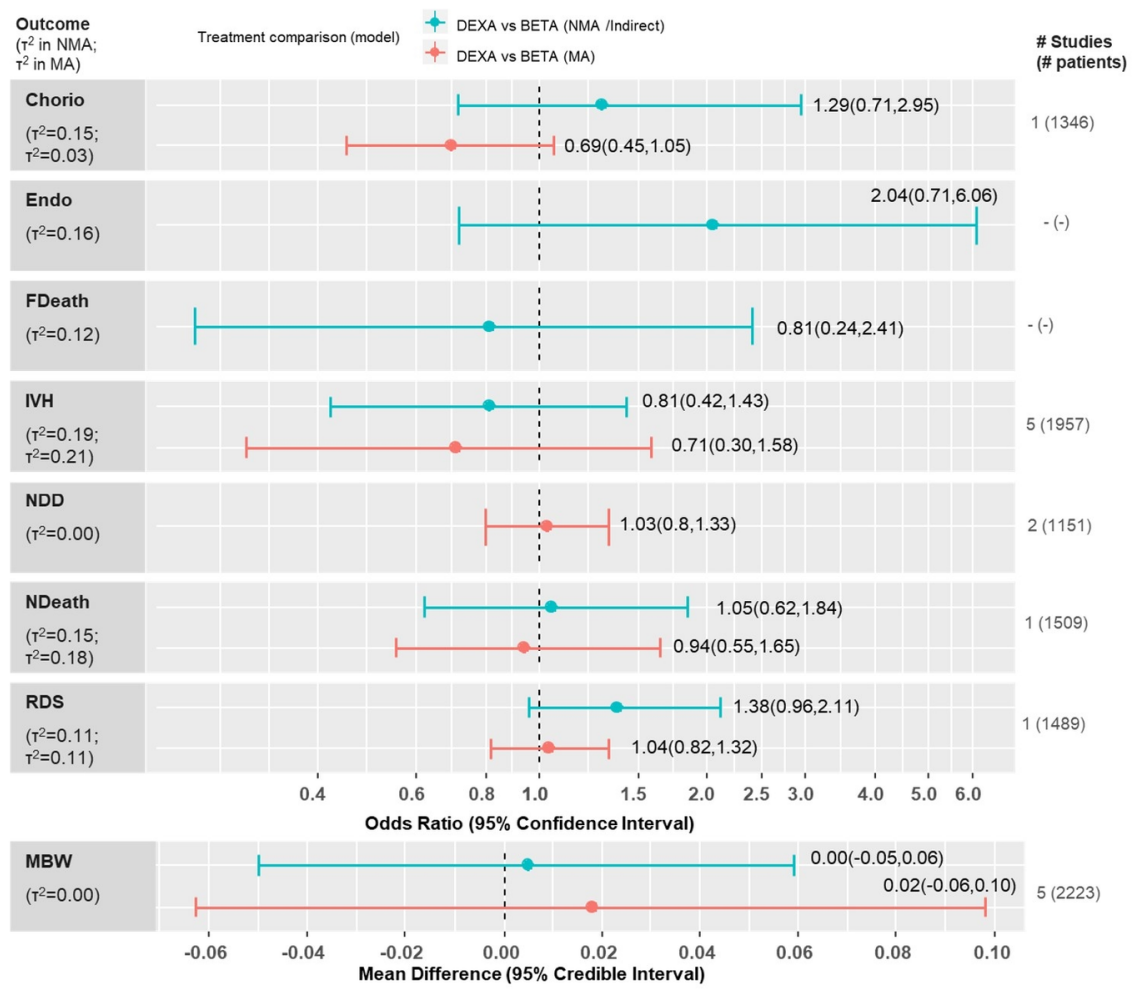

